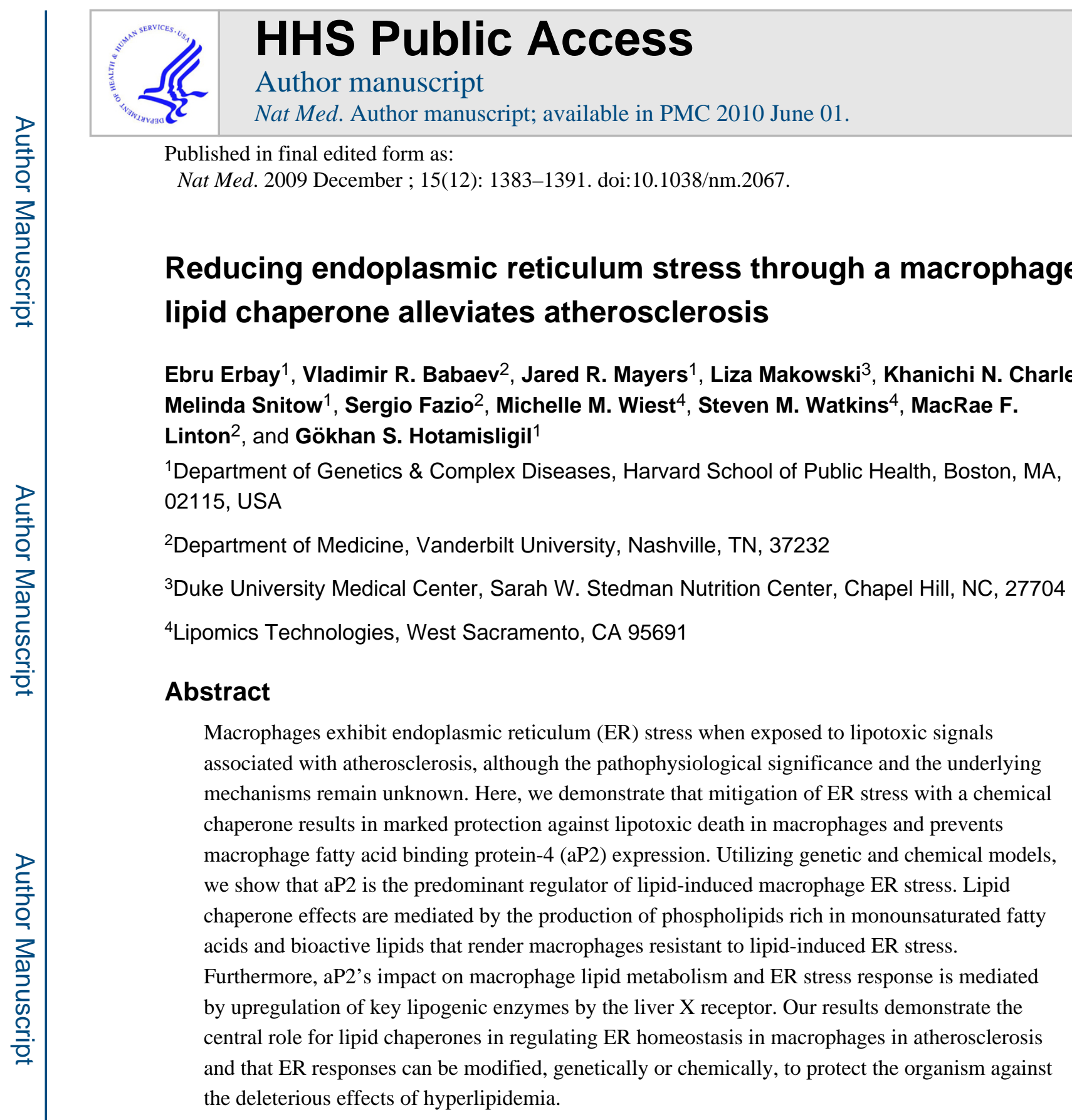

Published in final edited form as:

Nat Med. 2009 December ; 15(12): 1383-1391. doi:10.1038/nm.2067.

\title{
Reducing endoplasmic reticulum stress through a macrophage lipid chaperone alleviates atherosclerosis
} \author{
Melinda Snitow ${ }^{1}$, Sergio Fazio ${ }^{2}$, Michelle M. Wiest ${ }^{4}$, Steven M. Watkins ${ }^{4}$, MacRae F. \\ Linton $^{2}$, and Gökhan S. Hotamisligil ${ }^{1}$ \\ ${ }^{1}$ Department of Genetics \& Complex Diseases, Harvard School of Public Health, Boston, MA,
}

\begin{abstract}
Macrophages exhibit endoplasmic reticulum (ER) stress when exposed to lipotoxic signals associated with atherosclerosis, although the pathophysiological significance and the underlying mechanisms remain unknown. Here, we demonstrate that mitigation of ER stress with a chemical chaperone results in marked protection against lipotoxic death in macrophages and prevents macrophage fatty acid binding protein-4 (aP2) expression. Utilizing genetic and chemical models, we show that aP2 is the predominant regulator of lipid-induced macrophage ER stress. Lipid chaperone effects are mediated by the production of phospholipids rich in monounsaturated fatty acids and bioactive lipids that render macrophages resistant to lipid-induced ER stress. Furthermore, aP2's impact on macrophage lipid metabolism and ER stress response is mediated by upregulation of key lipogenic enzymes by the liver $\mathrm{X}$ receptor. Our results demonstrate the central role for lipid chaperones in regulating ER homeostasis in macrophages in atherosclerosis the deleterious effects of hyperlipidemia.
\end{abstract}

\footnotetext{
Users may view, print, copy, download and text and data- mine the content in such documents, for the purposes of academic research, subject always to the full Conditions of use: http://www.nature.com/authors/editorial_policies/license.html\#terms

To whom correspondence should be addressed: Gökhan S. Hotamisligil, M.D., Ph.D., Department of Genetics and Complex Diseases, Harvard-Massachusetts Institute of Technology Broad Institute, Harvard School of Public Health, Boston, MA 02115, Fax: 617432 1941, Phone: 617432 1950, ghotamis@hsph.harvard.edu.

CONTRIBUTIONS

E.E. developed the hypothesis, designed and performed the bulk of the experiments, analyzed all data and wrote the manuscript; V.R.B. contributed to the in vivo studies; J.R.M. contributed to the in vitro studies and conducted data analysis, K.N.C. and M.S contributed to the in vitro studies; L.M., M.M.W, S.M.W. contributed to the analysis of lipidomic data, S.F. and M.F.L. contributed to the analysis of in vivo data and assisted with writing; and G.S.H. developed the hypothesis, designed and analyzed all data, wrote the manuscript and supervised the project and the peer review process.
} 


\section{INTRODUCTION}

Organelle-mediated stress, particularly endoplasmic reticulum (ER) stress, has recently emerged as an important pathophysiological paradigm underlying chronic metabolic diseases $1-8$. In conjunction with its central role in protein synthesis, folding and transportation, the ER serves as a critical site for integrating cellular responses to stress9. The presence of misfolded proteins and other stresses lead to the activation of an adaptive program by the ER, known as the unfolded protein response (UPR), to reestablish equilibrium9. Initiation of the canonical UPR engages three distinct signaling branches mediated by pancreatic ER kinase (PERK), inositol-requiring transmembrane kinase/ endonuclease 1 (IRE1) and activating transcription factor 6 (ATF6). The UPR is also linked to the activation of stress kinases such as the c-Jun N-terminal kinase (JNK). The combined action of these pathways leads to inhibition of protein translation, stimulation of protein degradation, and the production of chaperone proteins resulting in either recovery of ER function or cell death10.

In obesity, activation of the UPR in metabolic tissue contributes to insulin resistance, at least in part through IRE-1-dependent, JNK-1-mediated inhibition of insulin action5. Promotion of ER stress by genetic X-box binding protein 1 (XBP-1) haploinsufficiency, which functions in the UPR-induced transcriptional program, also leads to systemic insulin resistance, while alleviation of ER stress by chemical or molecular chaperones protects mice against insulin resistance and type 2 diabetes5-7. Activation of ER stress response pathways is also a characteristic of lipid-laden macrophages in atherosclerotic lesions in mice and humans and is proposed to play a role in plaque vulnerability and acute cardiac death3,4,8. However, the role of ER stress in macrophages and cardiovascular disease remains obscure and it is unknown whether the modulation of ER stress pathways could alter the function and survival of macrophages and the course of atherosclerosis. Moreover, it is unclear how accumulation of excess lipids in macrophages can engage the ER stress response pathways. Despite continuing debate, it is likely that the biological effects of toxic lipids such as those prevalent in dyslipidemia are signaled through specific pathways rather than lipotoxicity representing a non-specific demise of cellular function and viability. Several pieces of evidence suggest a connection between lipid metabolism and the UPR. For example, XBP-1 plays a role in ER phosphatidylcholine synthesis and endomembrane expansion, and has been linked to transcriptional regulation of several lipogenic genes in the liver11,12. ER stress can induce lipogenesis and promote hepatic steatosis12-15. On the other hand, inhibition of phospholipid synthesis or increasing phospholipase activity exacerbates ER stress responses and sphingolipid levels can influence ER function16,17. Additionally, ER stress was identified as a mechanism driving free cholesterol-induced cell death, in a model of cholesterol loading3. Hence, it is possible that the ER may serve as an important target organelle that senses stresses related to lipid status and exposure10. However, the signaling networks linking ER function, lipid metabolism and the physiological outcomes are not known.

Cellular lipid metabolism and reception of lipid signals are regulated by cytosolic lipid chaperones, particularly fatty acid binding protein-4 (aP2), which exhibit profound effects on chronic metabolic diseases and whose function is relevant to human disease 10,18,19-23. 
The dramatic impact of aP2 on atherosclerosis is related exclusively to its action in the macrophages although the underlying mechanisms are not fully resolved22. The fact that macrophage aP2-deficiency can mediate protection from atherosclerosis in the setting of severe dyslipidemia raised the possibility that lipid chaperones may be a link between toxic lipids and organelle stress in macrophages.

Here, we explored the mechanisms related to lipotoxic macrophage ER stress and the impact of ER dysfunction on atherosclerosis utilizing a chemical chaperone and lipid chaperonedeficient mouse model. We demonstrate that mitigation of ER stress is protective against atherosclerosis and that $\mathrm{aP} 2$ is an obligatory intermediate for macrophage ER stress responses to lipids.

\section{RESULTS}

\section{I: Blocking macrophage ER stress and atherosclerosis}

The chemical chaperone, 4-phenyl butyric acid (PBA) can alleviate ER stress, and hence, provides an experimental opportunity to approach the role of ER stress in atherosclerosis7,24. We first tested whether PBA can alter ER stress induced in macrophages upon exposure to saturated fatty acids 25,26 . Treatment with palmitate (PA) induced ER stress in macrophages as determined by phosphorylation of PERK (P-PERK) and eukaryotic translation initiation factor $2 a(P-e I F 2-a)$. However, co-treatment with PBA resulted in essentially complete protection against PA-induced ER stress (Fig.1a). PBA treatment also suppressed PA-induced splicing of XBP-1 (sXBP-1) and C/EBP homologous protein (CHOP) expression, two elements of the UPR-induced transcriptional program (Fig. $1 b, c)$. Since saturated fatty acids or modified lipoproteins can induce apoptotic pathways we next asked whether modifying ER stress in this setting could prevent lipotoxic death in macrophages27. Treatment with PBA resulted in marked protection against PA-induced apoptosis in macrophages, as determined by TUNEL assays (Fig.1d and Supplementary Fig. 1a). These results demonstrate that PBA can protect cultured macrophages against lipidinduced ER stress and apoptosis in vitro.

Next, to elucidate whether PBA could mitigate ER stress in atherosclerotic lesions in vivo, we analyzed ER stress indicators and apoptosis in serial sections from aortic sinuses of atherosclerotic mice briefly treated with PBA. Six-week-old, male, $A p o E^{-/-}$mice were fed with a Western diet for 8 weeks, and given daily doses $\left(10 \mathrm{mg} \mathrm{kg}^{-1}\right.$ or $\left.100 \mathrm{mg} \mathrm{kg}^{-1}\right)$ of PBA or vehicle during the final 2 weeks. Examination of the sections of the aortic sinus for proximal lesions demonstrated that $A p o E^{-/-}$mice receiving PBA showed a dose-dependent reduction (9\%, NS and 32\%, p $<0.05$, respectively) in atherosclerosis (Fig.1e). At this early stage of atherosclerosis, en face analysis was similar between the groups (Supplementary Fig.1b). Of note, suppression of macrophage ER stress and reduction in vascular lesions by PBA treatment occurred in the absence of any impact on lipids, lipoprotein profiles, glucose and insulin levels in the circulation and body weight ( $30.6 \pm 0.4 \mathrm{~g}$ vs. $29.5 \pm 0.6 \mathrm{~g} ; \mathrm{p}=0.19$ ) (Supplementary Fig.1c-f and data not shown). Hence, this relatively short treatment period provided a suitable experimental design to examine the status of ER stress indicators and apoptosis in atherosclerotic lesions without significant changes in systemic metabolic parameters, insulin sensitivity or dramatic alterations in total burden of lesions that occurs 
with longer PBA treatment (data not shown)7,24. Immunohistochemical analysis revealed that all mice developed early lesions that predominantly contained macrophages and macrophage-derived foam cells (shown by staining with monocyte/macrophage-specific antibody, MOMA-2) (Fig.1f). There was no significant reduction in total macrophage area upon PBA treatment despite reduction in lesion size (macrophage area in control: $123097 \pm 13711$ and in PBA treatment: $170545 \pm 30249 \mu \mathrm{m}^{2}$ ). The atherosclerotic lesions of $A p o E^{-/-}$(control) mice stained positive for the ER stress markers, P-eIF2- $\mathrm{a}$ and P-PERK in macrophage dense areas (Fig.1f). In contrast, P-eIF2- $\alpha$ and P-PERK staining was significantly diminished in atherosclerotic lesions of mice treated with PBA (Fig.1f). Immunofluorescent staining revealed a 44\% reduction in ATF3 expression ( $\mathrm{p}<0.05)$ and a $53 \%$ reduction in P-eIF2- $\mathrm{a}$ expression $(\mathrm{p}<0.05)$ in the macrophage-dense areas of the lesions following PBA treatment (Fig.1g and Supplementary Fig.1g). We also examined the extent of apoptosis in these atherosclerotic lesions using TUNEL staining. Atherosclerotic lesions from control mice contained abundant apoptotic cells whereas lesions from PBA treated mice exhibited significant reduction in apoptotic cells in a dose dependent manner ( $29 \%$ and $42 \%$, respectively; $\mathrm{p}<0.05$ in the $100 \mathrm{mg} \mathrm{kg}^{-1}$ dose) (Fig. $1 \mathrm{~h}$ ). These results demonstrate that PBA treatment leads to marked reduction in macrophage ER stress and apoptosis in atherosclerotic lesions in vivo indicating that improvement of ER chaperoning function can protect against the deleterious effects of toxic lipids in promoting atherosclerotic lesions.

\section{II: Regulation of lipid-induced ER stress by a lipid chaperone}

It has been shown that aP2-deficiency in macrophages protects against atherosclerosis despite a highly unfavorable lipid profile22,28,29. Since, toxic lipids fail to trigger ER stress in the presence of PBA, we investigated whether lipid chaperoning activity is linked to ER stress and PBA activity in macrophages. Notably, aP2 immunostaining was significantly suppressed in vascular lesions of $A p o E^{-/-}$mice treated with PBA when compared to vehicle treated animals $(71 \%, \mathrm{p}<0.05)$ (Fig.2a,b). Furthermore, PA induced rapid and marked upregulation of aP2 protein, but not $a P 2$ mRNA levels (Fig.2c). Induction of aP2 by PA was prevented upon co-treatment with PBA (Fig.2c). Taken together, these results indicate that aP2 expression is directly related to lipid-induced ER stress and strongly inhibited by PBA in macrophages in vitro and in vivo. This observation led us to ask whether aP2 regulates ER responses to lipid stress in macrophages. Treatment of wild type (WT) macrophages with long-chain saturated fatty acids such as PA or stearate (STE), but not their monounsaturated counterparts, led to ER stress as judged by the robust phosphorylation of PERK and eIF2-a, activation of JNK, and induction Ddit3 and $s X B P-1$ expression (Fig.2d,e and Supplementary Fig.2b,d and g). However, PA failed to induce ER stress in aP2-deficient $\left(a 2^{-/-}\right)$ macrophages (Fig.2d,e and Supplementary Fig.2g). In these cell lines, there were no alterations in PA uptake (Supplementary Fig.2e). The $a P 2^{-/-}$cells also maintained the ability to respond to tunicamycin, an inhibitor of protein glycosylation that leads to ER stress, indicating that they do not suffer from a general defect in mounting ER stress responses (Fig.2d,e).

We then compared ER stress responses in $a P 2^{-/-}$macrophages reconstituted with a lipidbinding mutant (LM) of aP2 (R126L, Y128F; $\left.a P 2^{-/} \mathrm{LM}\right)$ to those reconstituted with WT 
aP2 $\left(a P 2^{-/}\right.$REC) 30 . Under the conditions tested, WT- and LM-aP2 proteins were expressed at comparable levels to each other and did not lead to any alterations in lipid uptake (Supplementary Fig.2e and data not shown). Reconstitution of WT-aP2 rendered $a P 2^{-/-}$macrophages responsive to PA as indicated by the induction of P-PERK, P-eIF2- $a$ and JNK activity, while $a P 2^{-/}$LM macrophages remained markedly resistant to PAinduced ER stress (Fig.2d). PA also induced the expression of Ddit 3 and sXBP-1 in WT but not in $a P 2^{-/-}$macrophages (Supplementary Fig.2g and Fig.2e). In $a P 2^{-/-}$macrophages, reconstitution of aP2 restored the induction of UPR target genes by PA (Fig.2d,e and Supplementary Fig.2g). Consistent with their ER stress-resistant phenotype, $a 2^{-/-}$ macrophages were also significantly protected against PA-induced apoptosis as indicated by suppression of caspase-3 activity and PARP cleavage (Fig.2f and Supplementary Fig.2h). Reconstitution of WT-aP2 into the $a P 2^{-/-}$macrophages rendered these cells susceptible to lipid-induced apoptosis whereas cells expressing LM-aP2 remained refractory to apoptosis, demonstrating the requirement for the lipid binding activity of aP2 in regulating ER stress responses (Fig.2f and Supplementary Fig.2h).

Next, we exposed macrophages to free cholesterol (FC) loading in order to examine ER stress responses in another setting of lipotoxicity associated with the pathogenesis of atherosclerosis4. In WT, but not $a P 2^{-/-}$macrophages, FC induced ER stress as indicated by the induction of P-PERK and P-eIF2-a, sXBP-1, and JNK activity (Fig.2g). These observations were also independent of compromised cholesterol uptake; in fact, $a \mathrm{P}^{-/-}$ macrophages exhibit increased cholesterol influx31. Responsiveness to FC-induced ER stress was restored in $a P 2^{-/-}$macrophages, upon reconstitution of aP2, demonstrating that aP2 mediates FC-induced ER stress in macrophages (Fig.2g). Furthermore, $a P 2^{-/-}$ macrophages were resistant to FC-induced induced apoptosis, determined by activation of caspase 3 and cleavage of PARP, that normally occurs in FC-treated WT macrophages (Supplementary Fig.2i)3. Taken together our results show that aP2 is necessary for toxic lipids to trigger ER stress and apoptosis in macrophages.

\section{III: Regulation of macrophage ER stress by aP2 in vivo}

We next investigated whether aP2-deficiency in mice can modulate ER stress responses in vascular lesions in vivo. The $a \mathrm{P}^{-/-}$mouse model provides an ideal setting to examine the link between macrophage ER stress and atherosclerosis since aP2-deficiency does not alter the hyperlipidemia or any other metabolic parameters in the $A p o E^{-/-}$background, and furthermore, aP2's impact on atherosclerosis is predominantly, if not completely, mediated by its action in the macrophages22. The early stage atherosclerotic plaques from $A p o E^{-/-}$ mice showed induction of ER stress as indicated by elevated P-PERK, P-eIF2-a, and Ddit3 mRNA in the infiltrating macrophages within the lesions (Fig.3a). Notably, the vascular lesions in the $a \mathrm{P}^{-/-} \mathrm{ApoE^{-/- }}$ mice were essentially devoid of staining for markers of ER stress (Fig.3a). Quantitative analysis of ER stress by immunofluorescent staining demonstrated a significant reduction in P-eIF2- $\alpha$ and ATF3 (55\% and 67\%, respectively; $\mathrm{p}<0.05$ ) expression in macrophage-rich areas of the lesions (Fig.3b,c). Furthermore, TUNEL assays demonstrated a significant reduction in the number of apoptotic macrophages in lesions of $a P 2^{-/-} \mathrm{ApoE}^{-/-}$mice compared to $\mathrm{ApoE}^{-/-}$animals $(7.8 \%$ and $18.4 \%$, respectively; p $<0.05$ ) (Fig.3d,e and Supplementary Fig.3a). This confirmed the critical role 
of aP2 in mediating macrophage ER stress response to toxic lipids in vivo, similar to its actions in cultured macrophages in vitro.

The impact of genetic aP2-deficiency can be mimicked by a specific chemical inhibitor for aP2 (aP2-i) in vitro and in vivo29. Treatment of WT macrophages with aP2-i also led to marked protection against PA-induced ER stress as assessed by diminished P-PERK, PeIF2-a, Ddit3 and sXBP-1 expression, without any effects on fatty acid uptake (Fig.3f,g and Supplementary Fig.3b). Treatment with aP2-i also reduced ATF3 expression in macrophagedense areas of the plaques in $\mathrm{ApoE}^{-/-}$mice in vivo, without altering hyperlipidemia (Fig. $3 \mathrm{~h}) 29$. These results clearly demonstrate that genetic or chemical ablation of aP2 protects macrophages from ER stress in vivo in the context of hypercholesterolemic atherosclerosis.

\section{IV: Mechanisms linking aP2 to macrophage ER stress}

In order to identify the metabolic pathways that control the observed tolerance to toxic lipids, we next studied the impact of aP2 on macrophage lipid composition and metabolism. We analyzed the profiles of individual fatty acids (FA) in macrophages in a systematic manner using high resolution, quantitative lipidomic analysis. $a P 2^{-/-}$macrophages contained elevated levels of monounsaturated fatty acids (MUFA), indicating that a greater proportion of the lipids in these cells were produced de novo (Supplementary Table 1). Furthermore, we saw evidence of increased delta 9 desaturase/steaoryl CoA desaturase $(\triangle 9 \mathrm{D} / \mathrm{SCD})$ activity in $a P 2^{-/-}$macrophages, reflected in the elevated $\mathrm{C} 16: 1 \mathrm{n} 7 / \mathrm{C} 16: 0$; C14:1n5/C14:0, and C18:1n9/C18:0 ratios of FA present in various lipid classes (Fig.4a and Supplementary Table 1). Enhancement of de novo lipogenesis in $a P 2^{-/-}$macrophages resulted in marked increase in $\mathrm{C} 16: 1 \mathrm{n} 7$ levels and its direct elongation product, $\mathrm{C} 18: \ln 7$ in addition to a modest elevation in C14:1n5 and C18:1n9 (Fig.4b and Supplementary Table 1). The reconstitution of aP2 dramatically shifted the FA profile from one of active de novo synthesis, with enhanced desaturase activity, to high elongase activity with little desaturase action, demonstrating that macrophage de novo lipogenesis is strongly regulated by aP2 (Fig.4a,b and Fig.5a).

We next asked which lipid classes are enriched by these newly synthesized fatty acids and determined the distribution of all major classes of lipids. When compared to WT macrophages, $a P 2^{-/-}$macrophages had elevated phospholipids (PL;\%138), triglycerides (TG;140\%), diacylglycerol (DG;143\%) and free fatty acid (FFA;224\%) concentrations and lower levels of cholesterol esters (CE;79\%) (Fig.4c,d). Reconstitution of aP2 suppressed PL levels by $31 \%$ and dramatically increased CE concentrations by $197 \%$, demonstrating a crucial role for aP2 in the regulation of macrophage phospholipid and cholesterol production (Fig.4c,d). The marked increase in the phospholipid-to-cholesterol ratio seen in the $a P 2^{-/-}$ macrophages suggests that one potential way aP2 can modulate stress responses to toxic lipids may be through alteration in membrane lipid composition and metabolic properties.

The results of lipidomic analysis implicated regulation of de novo lipogenesis and desaturation, a rate-limiting step catalyzed by SCD, as a potential mechanism underlying the aP2-driven compositional changes in macrophages (Fig.5a). The desaturase activity of SCD converts saturated FA to MUFA which are then incorporated into phospholipids, triglycerides, and cholesterol esters32,33. Indeed, $a P 2^{-/-}$macrophages are enriched with 
MUFA, most significantly in C16:1n7-palmitoleate and its direct elongation product, C18:1n7, across all major lipid classes (Fig.4b and Supplementary Table 1). Consistently, $\mathrm{SCD}-1$ expression in $a 2^{-/-}$macrophages was $\sim 50$ fold-higher than that of WT controls (Fig.5b). Next, we examined aP2-regulated SCD-1 expression in macrophages in vivo and found that its expression was markedly upregulated in the peritoneal macrophages isolated from mice treated with aP2-i (Fig.5c). Fatty acid synthase (Fasn) expression was also significantly elevated in $a 2^{-/-}$macrophages and induced upon aP2-i treatment in vivo (data not shown). Collectively, these data indicate that aP2 action in macrophages is linked to the regulation of key enzymes involved in the de novo synthesis and desaturation of fatty acids.

To examine the impact of aP2-regulated SCD-1 activity in the resistance to ER stress in $a P 2^{-/-}$macrophages, we took two distinct but related approaches. First, we asked whether $\mathrm{C} 16$ :1n7-palmitoleate, a product of de novo lipogenesis elevated in $a P 2^{-/-}$macrophages, could modify ER responses to lipids34. Strikingly, we found that WT macrophages pretreated with C16:1n7-palmitoleate became resistant to PA-induced ER stress and apoptosis, but not to tunicamycin-induced ER stress, determined by the P-PERK, P-eIF2-a, cleaved PARP and Ddit 3 and $s X B P-1$ levels. This pattern induced by C16:1n7-palmitoleate was highly reminiscent of genetic or chemical aP2-deficiency (Fig.5d,e). Oleate, another FA product of desaturation, which is not regulated to the same extent as palmitoleate in $a P 2^{-/-}$ macrophages, could also protect against PA or STE- induced ER stress (Supplementary Fig. $2 b-d)$.

Second, we utilized a siRNA-mediated approach to significantly deplete SCD-1 protein and activity in $a P 2^{-/-}$macrophages (Fig.5f). Sensitivity to PA-induced ER stress and apoptosis was re-established in the $S C D-1$ siRNA treated $a 2^{-/-}$macrophages, but not in scrambled (control) siRNA treated cells, as evidenced by increased P-PERK and P-eIF2- $a$, active caspase 3 and cleaved PARP levels (Fig.5g). These results demonstrate that aP2-mediated regulation of SCD-1 activity is causally linked to lipid-induced ER stress responses in macrophages.

Unraveling the molecular mechanisms by which aP2 regulates SCD-1 is important for understanding how lipid-stress signals impinge on the lipid synthetic pathways. Both $S C D-1$ and Fasn are direct transcriptional targets of the nuclear receptor LXR (LXR-responsive elements, LXRE, are located on Fasn, between positions -669 and -665, and SCD-1, between positions -1263 and -1248) (Fig.6a)35,36. Thus, we analyzed whether LXR activity is altered in aP2-deficient macrophages by utilizing an LXRE-driven reporter. The $a P 2^{-/-}$macrophages displayed a significant elevation in stimulated LXR activity when compared to control, $a P 2^{-/}$REC cells (Fig.6b). These results indicate that aP2 negatively regulates LXR activity in WT macrophages. Consistently, we observed increased expression of LXR target genes Abcal, Abcgl and CD51 (AIM) in $a P 2^{-/-}$macrophages (Supplementary Fig.4a-c)37-39. Further examination revealed marked elevation of NrIh3 (LXR-a) mRNA and protein levels in the absence of aP2, while NrIh2 (LXR- $\beta$ ) expression remained unchanged between the genotypes (Fig.6c and Supplementary Fig.4d,e). Hence, the LXR- $a$ expression appeared to be the main driver of the alterations seen in LXR target gene expression. In order to definitively link LXR-a activity to $S C D-1$ regulation, we next 
suppressed $\mathrm{Nr} \mathrm{h} 3 \mathrm{3}$ expression in $a P 2^{-/-}$macrophages using a siRNA-mediated knock down approach (Supplementary Fig.5a). Reduction of $\mathrm{Nrlh} 3$ expression had only a partial effect on the expression genes regulated by both LXR-a and $-\beta$, such as Abcal and Abcgl, but generated a profound effect on the expression of an LXR-a exclusive target gene, CD51 (AIM) (Supplementary Fig.5b-d)37-39. The reduction in LXR-a also led to significant inhibition of both Fasn and $S C D-1$ mRNA levels, demonstrating that LXR-a is mainly responsible for the upregulation of these genes in the absence of aP2 (Fig.6d and Supplementary Fig.5e). Suppression of LXR-a also restored sensitivity to PA-induced ER stress and apoptosis as determined by induction of P-PERK and P-eIF2- $a$ and cleaved PARP levels in $a P 2^{-/-}$macrophages (Fig.6e). To validate these links in a genetic setting, we examined lipid-induced ER stress response in primary peritoneal macrophages derived from mice with $a P 2$ and $N r l h 3$ combined genetic deficiency. The expression of Fasn and $S C D-1$ was also markedly down-regulated in $a P 2^{-/-} \mathrm{Nr}_{\mathrm{h}} 3^{-/-}$macrophages compared to $a P 2^{-/-}$cells (Supplementary Fig.6b,c)40. The expression of CD51 was profoundly suppressed in double mutant cells, while Abcal and $A b c g l$ were only partially affected (Supplementary Fig.6d-f)37-39. Consequently, the protection against PA-induced ER stress was also lost in the $a \mathrm{P}^{-/-} \mathrm{Nr} / h 3^{-/-}$cells as determined by P-PERK, P-eIF2- $\mathrm{a}$ and cleaved PARP induction (Fig.6f). Consistently, treatment of WT macrophages with a specific LXR agonist, T0901317, promoted resistance to lipid-induced ER stress, similar to aP2-deficiency (Supplementary Fig.5f). These results illustrate that LXR-a is responsible for the upregulation of SCD-1 in the absence of aP2 and provides one crucial mechanism for how lipid stress signals may impinge on macrophage lipid metabolism and ER stress (Fig.6g).

\section{DISCUSSION}

Macrophages are particularly vulnerable to lipid-induced toxicity and contribute to the pathogenesis of several metabolic derangements where exposure to lipids is increased, such as the foam cells in hypercholesterolemic atherosclerosis and in adipose tissue-associated macrophages in obesity2,10,41. Previous studies have shown expression of UPR markers in macrophages infiltrating the atherosclerotic lesions of both mice and humans 4,8 . These findings are complemented by in vitro studies demonstrating that accumulation of free cholesterol leads to apoptosis via activation of ER stress in macrophages3. Elevation of ER stress-associated macrophage apoptosis has been proposed to contribute to advanced atherosclerotic lesions in macrophages defective in insulin signaling42. While these findings have sparked interest, demonstration of a link between macrophage ER stress and atherogenesis in vivo and the mechanisms integrating lipid signals to ER function in macrophages has been challenging. Hence, modulation of ER stress, especially upstream of the apoptotic execution pathways, becomes critical in understanding the extent of its contribution to the pathogenesis of atherosclerosis. The data presented in this paper provide insight into these critical questions. First, we demonstrate that the lipid chaperone aP2 is an obligatory mediator coupling toxic lipids to ER stress in macrophages in vitro and in vivo. Second, we show that alleviating ER stress, either by the use of a chemical chaperone or through the inhibition of a lipid chaperone, provides significant protection against macrophage ER stress, cell death, and atherosclerosis. 
The surprising and striking upregulation of aP2 by saturated fatty acids and downregulation by PBA in macrophages led to the uncovering of a previously unknown function of the lipid chaperones in mitigating ER stress in macrophages in vitro and in atherosclerotic lesions in vivo. The observations place aP2 as a central modulator of lipid-induced ER stress responses-a process for which there had been previously little mechanistic insight. It is also established that the role of macrophage aP2 action on atherosclerosis is not related to other metabolic alterations, such as changes in insulin sensitivity or dyslipidemia, and is therefore intrinsic to macrophages 22,43 . Similarly, the treatment with PBA dose used in this study, and as reported earlier, does not yield significant metabolic alterations7. Therefore, our present findings unravel a mechanism by which aP2 could mediate its antiatherosclerotic effects through modulating the UPR, helping to clarify a much sought after but very challenging aspect of lipid chaperone biology in macrophages and in atherosclerosis4,8. Finally, our findings offer tools to modulate ER stress responses associated with dyslipidemia in vitro and in vivo that may facilitate therapeutic applications including that of aP2.

Dramatic resistance to ER stress can be achieved by blocking aP2 action, which is dependent on SCD-1 activity. aP2 prevents enrichment of macrophages in desaturation products such as $\mathrm{C} 16: 1 \mathrm{n} 7$-palmitoleate, a molecule which provides relief from lipid-induced ER stress, in addition to its other reported beneficial endocrine effects34. It will be interesting to determine whether $\mathrm{C} 16: 1 \mathrm{n} 7$ supplementation or a diet enriched with palmitoleate could confer resistance to macrophage ER stress and reduction in atherosclerosis in future studies. In this study, we showed that enhanced LXR-a activity in $a P 2^{-/-}$macrophages drives Fasn and $S C D-1$ transcription and resistance to lipotoxic ER stress and identified the transcriptional mechanism underlying this function of aP2. These observations also raise the possibility of a specific link between the nuclear hormone receptor LXR-a and ER stress responses. Overall, this study demonstrates that de novo fatty acid synthesis and desaturation can be highly beneficial, if not essential, for defending ER function when macrophages are exposed to toxic lipids. Accordingly, inhibition of SCD-1 in the whole body may not have great therapeutic prospects due to adverse metabolic effects seen in the macrophages and pancreatic $\beta$ cells, although elevated liver $\Delta 9 \mathrm{D}$ activity has been linked to obesity and diabetes10,44. Indeed, systemic inhibition of SCD-1 leads to severe atherosclerosis despite protection from obesity and hepatosteatosis 45 . Similarly, activation of LXR in the whole body leads to undesirable metabolic side effects, particularly in the liver. On the other hand selective upregulation of LXR and SCD-1 activity in macrophages and adipocytes, and downregulation in liver may be an optimal strategy and yield the most beneficial overall metabolic outcome, including protection against diabetes and atherosclerosis 29 .

We unraveled a mechanism by which aP2 could mediate its anti-atherosclerotic effects, at least in part, through modulating the UPR. It is important to note that our findings do not exclude other LXR regulated macrophage responses as additional contributors to aP2's impact on macrophage function and atherosclerosis. Since the most significant upregulation seen in the $a P 2^{-/-}$macrophages is that of $S C D-1$ and $C D 51$ genes and knocking down SCD-1 alone is sufficient to re-establish sensitivity to lipid-induced ER dysfunction in the 
$a P 2^{-/-}$macrophages, LXR-mediated lipogenesis pathways are likely to dominate the aP2deficient phenotype related to lipotoxic ER stress. Yet, the impact of aP2 or LXR on inflammatory functions of macrophages may also play a role in preventing atherosclerosis. aP2 expression is limited to macrophages and dendritic cells in normal conditions as well as in atherosclerotic lesions (Fig.2B and data not shown)10,22,46,47. While aP2 does not alter antigen presentation, studies have shown that it can affect $\mathrm{T}$ cell priming and cytokine production19,46,47. Moreover, ER stress responses and inflammation are integrated at several levels and modulate each other; inflammation can compromise ER function and ER stress can promote inflammation10,. The links between inflammatory pathways and ER stress are of great interest in chronic metabolic disease and understanding the intricate links between lipid metabolism and the immune system and identification of molecular targets like aP2 at this interface are critical for developing effective therapeutics against the metabolic disease cluster.

In conclusion, we uncovered a previously unknown function for aP2 in lipid-induced ER stress signaling in macrophages, while addressing the essential role of ER stress in vascular disease progression (Fig.6g). The ability to defend against the lipids disrupting ER function illustrates a novel metabolic adaptation capacity of macrophages that is governed by the lipid chaperones. aP2 in particular, and perhaps cytosolic lipid chaperones in general, could function as a molecular sensor for fatty acids and as a central coordinator of metabolic-ER stress. Since aP2-deficiency can alleviate the ER stress that occurs during atherosclerosis, similar to the actions of a chemical chaperone, our findings offer insights into the detrimental role of macrophage ER stress in atherosclerosis and the benefits of addressing this target to treat cardiovascular disease.

\section{MATERIALS AND METHODS}

\section{Mice, immunhistochemistry and quantification of arterial lesions}

Colonies for $a \mathrm{P}^{-/-} \mathrm{ApoE}^{-/-}$, $\mathrm{ApoE}^{-/-}$and $a \mathrm{P}^{-/-} \mathrm{Nr} \mathrm{h} 3^{-/-}$mice on the C57BL/6 background were established in our facilities. $\mathrm{Nr}_{\mathrm{fh}} 3^{-/-}$mice used in this study were previously published40. Harvard Medical Area Standing Committee on Animals approved the animal handling procedures. Mice were fed with Western diet for 16 weeks as previously described29. After sacrifice, aortas were flushed through the left ventricle and dissected as described earlier29. To detect macrophages in arterial lesions, $5 \mu \mathrm{m}$ serial cryosections of the proximal aorta were fixed in acetone and incubated with antibodies following manufacturer's recommendations and previously established protocols 48 . The sections were incubated with biotinylated secondary antibodies and then with alkaline phosphatase-labeled ABC (Vector Lab). Lesion areas were quantified using Imaging System KS 300 (2.0;

Kontron Electronik GmbH). For immunofluorescence stainings, Alexa Fluor 488- and Alexa Fluor 647-conjugated secondary antibodies were applied after an overnight incubation of the lesions with the primary antibody. DAPI was used for counterstaining. The mean fluorescent intensity was measured for each corresponding ER stress marker in the macrophage-dense areas using Auxiovision 4.6 software $(\mathrm{n} \geq 3)$. For TUNEL analysis, serial the sections were pretreated with 3\% citric acid, fixed in $4 \%$ paraformaldehyde and stained using an in situ cell death detection AP kit (Roche). After visualization of alkaline phosphatase with Fast 
Red TR/Naphthol AS-NX substrate (Sigma), TUNEL-positive cells were counted ( $\mathrm{n}=18$ per aorta).

\section{Analysis of serum lipids}

Mice were fasted (4h) and the serum cholesterol and triglycerides were measured by conventional enzymatic methods using the reagents from Raichem and the SoftMax Pro5 software (Molecular Devices).

\section{Quantitative real-time polymerase chain reaction (qRT-PCR) and immunoblot analysis}

RNA was isolated from macrophages using RNeasy kit (Qiagen), cDNA was synthesized using iScript (Biorad) and qRT-PCR was performed using an ABI Thermocycler. Macrophage proteins were harvested and total protein content was assessed as previously described31. Equal amounts of total protein per sample were subjected to SDS-PAGE and Western blots were performed as described previously49.

\section{Cell culture, knock-down, TUNEL, caspase and reporter assays}

Unless otherwise indicated, bone-marrow derived mouse macrophage lines were used. Reconstitution experiments were carried out in bone-marrow derived macrophages that were immortalized29. When indicated, peritoneal macrophages derived by $4 \%$ thioglycolate elicitation were used. Macrophages were maintained in RPMI supplemented with $10 \%$ fetal bovine serum. For caspase 3/7 activity assays, cells were plated on 96 well plates, and upon reaching confluency, treated with the appropriate reagents to activate caspase activity over a time course. Caspase 3/7 activity was measured using the caspase 3/7-Glo kit (Promega) according to the manufacturer's instructions. For loss-of-function experiments, siRNAs were transfected into 80\% confluent macrophages using sImporter (Upstate) according to the manufacturer's instructions. For reporter assays, macrophages plated on 12-well plates, grown to $80 \%$ confluency, and transfected with the appropriate plasmids using Superfect

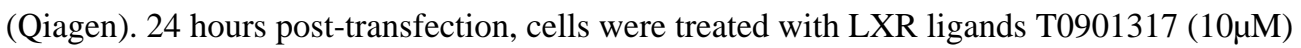
and 25 hydroxy cholesterol $(10 \mu \mathrm{M})$, or DMSO followed by luciferase assays using the DualLuciferase assays system (Promega) according to the manufacturer's instructions.

\section{Lipidomics}

Fatty acids (FA) were measured in 1 experiment $(\mathrm{n}=5)$ by Lipomics, Inc (Sacramento, CA) in each neutral lipid class CE, DAG, PL, FFA and TG. The lipids from plasma and tissues were extracted in the presence of authentic internal standards by the method of Folch et al. using chloroform:methanol (2:1 v/v). Individual lipid classes within each extract are separated by liquid chromatography (Agilent Technologies model 1100 Series)34. Each lipid class was trans-esterified in $1 \%$ sulfuric acid in methanol in a sealed vial under a nitrogen atmosphere at $100{ }^{\circ} \mathrm{C}$ for $45 \mathrm{~min}$. The resulting FA methyl esters were extracted from the mixture with hexane containing $0.05 \%$ butylated hydroxytoluene and prepared for gas chromatography by sealing the hexane extracts under nitrogen. Fatty acid methyl esters were separated and quantified by capillary gas chromatography (Agilent Technologies 6890) equipped with a $30 \mathrm{~m}$ DB-88MS capillary column (Agilent Technologies) and a flameionization detector. 


\section{Statistics}

One-way ANOVA was used to determine significance in lesion size differences. TUNEL assays: Statistical differences were determined by one-way ANOVA multiple comparisons versus the control group (Dunn's method) in Fig.1 and. the statistical differences in mean of TUNEL(+)/MOMA-2(+) lesion area between the groups were determined by the MannWhitney rank sum test in Fig.3. Lipidomic analyses: The distributions of each fatty acid within each lipid class were examined for extreme outliers or poor measurement. If a fatty acid was missing more than $30 \%$ of its observations, it was removed from further analysis. Initial statistical analysis included a one-way ANOVA to identify fatty acids within each lipid class that differed between the genotypes. Results of the one-way ANOVA were visualized using lipid class composition analysis. Direct comparisons between groups were made using a Wilcoxon rank test and visualized using bar plots.

\section{Supplementary Material}

Refer to Web version on PubMed Central for supplementary material.

\section{ACKNOWLEDGEMENTS}

This project has been supported by grants from National Institutes of Health to GSH DK DK52539 (to GSH), HL65405 (to MFL and GSH) and DK59637 (Lipid, Lipoprotein and Atherosclerosis Core of the Vanderbilt Mouse Metabolic Phenotype Center). EE is supported by the Ruth Kirschstein National Research Award. We are grateful to the members of the Hotamisligil lab, Jie Chen and Rebecca Bachman for their scientific input and contributions, to Aybike Onur for technical assistance, to Rebecca Foote and Kristen Gilbert for administrative support, to David Mangelsdorf (UT Southwestern) for TK-LXRE-X3luc reporter and $\mathrm{Nr}_{\mathrm{H}} \mathrm{h}^{-/-}$mice, and Alan Edgar (Fournier, France) for the ACAT inhibitor.

\section{REFERENCES}

1. Hotamisligil GS. Inflammation and metabolic disorders. Nature. 2006; 444:860-867. [PubMed: 17167474]

2. Gregor MF, Hotamisligil GS. Thematic review series: Adipocyte Biology. Adipocyte stress: the endoplasmic reticulum and metabolic disease. J Lipid Res. 2007; 48:1905-1914. [PubMed: 17699733]

3. Feng B, et al. The endoplasmic reticulum is the site of cholesterol-induced cytotoxicity in macrophages. Nat Cell Biol. 2003; 5:781-792. [PubMed: 12907943]

4. Tabas I. Consequences and therapeutic implications of macrophage apoptosis in atherosclerosis: the importance of lesion stage and phagocytic efficiency. Arterioscler Thromb Vasc Biol. 2005; 25:2255-2264. Epub 2005 Sep 2251. [PubMed: 16141399]

5. Ozcan U, et al. Endoplasmic reticulum stress links obesity, insulin action, and type 2 diabetes. Science. 2004; 306:457-461. [PubMed: 15486293]

6. Ozawa K, et al. The endoplasmic reticulum chaperone improves insulin resistance in type 2 diabetes. Diabetes. 2005; 54:657-663. [PubMed: 15734840]

7. Ozcan U, et al. Chemical chaperones reduce ER stress and restore glucose homeostasis in a mouse model of type 2 diabetes. Science. 2006; 313:1137-1140. [PubMed: 16931765]

8. Myoishi M, et al. Increased endoplasmic reticulum stress in atherosclerotic plaques associated with acute coronary syndrome. Circulation. 2007; 116:1226-1233. [PubMed: 17709641]

9. Ron D, Walter P. Signal integration in the endoplasmic reticulum unfolded protein response. Nat Rev Mol Cell Biol. 2007; 8:519-529. [PubMed: 17565364]

10. Hotamisligil GS, Erbay E. Nutrient sensing and inflammation in metabolic diseases. Nat Rev Immunol. 2008; 8:923-934. [PubMed: 19029988] 
11. Sriburi R, Jackowski S, Mori K, Brewer JW. XBP1: a link between the unfolded protein response, lipid biosynthesis, and biogenesis of the endoplasmic reticulum. J Cell Biol. 2004; 167:35-41. [PubMed: 15466483]

12. Lee AH, Scapa EF, Cohen DE, Glimcher LH. Regulation of hepatic lipogenesis by the transcription factor XBP1. Science. 2008; 320:1492-1496. [PubMed: 18556558]

13. Ferre P, Foufelle F. SREBP-1c transcription factor and lipid homeostasis: clinical perspective. Horm Res. 2007; 68:72-82. [PubMed: 17344645]

14. Oyadomari S, Harding HP, Zhang Y, Oyadomari M, Ron D. Dephosphorylation of translation initiation factor 2 alpha enhances glucose tolerance and attenuates hepatosteatosis in mice. Cell Metab. 2008; 7:520-532. [PubMed: 18522833]

15. Rutkowski DT, et al. UPR pathways combine to prevent hepatic steatosis caused by ER stressmediated suppression of transcriptional master regulators. Dev Cell. 2008; 15:829-840. [PubMed: 19081072]

16. Ramanadham S, et al. Apoptosis of insulin-secreting cells induced by endoplasmic reticulum stress is amplified by overexpression of group VIA calcium-independent phospholipase A2 (iPLA2 beta) and suppressed by inhibition of iPLA2 beta. Biochemistry. 2004; 43:918-930. [PubMed: 14744135]

17. Tessitore A, et al. GM1-ganglioside-mediated activation of the unfolded protein response causes neuronal death in a neurodegenerative gangliosidosis. Mol Cell. 2004; 15:753-766. [PubMed: 15350219]

18. Furuhashi M, Hotamisligil GS. Fatty acid-binding proteins: role in metabolic diseases and potential as drug targets. Nat Rev Drug Discov. 2008; 7:489-503. [PubMed: 18511927]

19. Shum BO, et al. The adipocyte fatty acid-binding protein $\mathrm{aP} 2$ is required in allergic airway inflammation. J Clin Invest. 2006; 116:2183-2192. [PubMed: 16841093]

20. Hotamisligil GS, et al. Uncoupling of obesity from insulin resistance through a targeted mutation in aP2, the adipocyte fatty acid binding protein. Science. 1996; 274:1377-1379. [PubMed: 8910278]

21. Uysal KT, Scheja L, Wiesbrock SM, Bonner-Weir S, Hotamisligil GS. Improved glucose and lipid metabolism in genetically obese mice lacking aP2. Endocrinology. 2000; 141:3388-3396. [PubMed: 10965911]

22. Makowski L, et al. Lack of macrophage fatty-acid-binding protein aP2 protects mice deficient in apolipoprotein E against atherosclerosis. Nat Med. 2001; 7:699-705. [PubMed: 11385507]

23. Cao H, et al. Regulation of metabolic responses by adipocyte/macrophage Fatty Acid-binding proteins in leptin-deficient mice. Diabetes. 2006; 55:1915-1922. [PubMed: 16804058]

24. Ozcan U, et al. Loss of the tuberous sclerosis complex tumor suppressors triggers the unfolded protein response to regulate insulin signaling and apoptosis. Mol Cell. 2008; 29:541-551. [PubMed: 18342602]

25. Borradaile NM, et al. Disruption of endoplasmic reticulum structure and integrity in lipotoxic cell death. J Lipid Res. 2006; 47:2726-2737. [PubMed: 16960261]

26. Lai E, Bikopoulos G, Wheeler MB, Rozakis-Adcock M, Volchuk A. Differential activation of ER stress and apoptosis in response to chronically elevated free fatty acids in pancreatic beta-cells. Am J Physiol Endocrinol Metab. 2008; 294:E540-E550. [PubMed: 18198352]

27. Schaffer JE. Lipotoxicity: when tissues overeat. Curr Opin Lipidol. 2003; 14:281-287. [PubMed: 12840659]

28. Tuncman G, et al. A genetic variant at the fatty acid-binding protein aP2 locus reduces the risk for hypertriglyceridemia, type 2 diabetes, and cardiovascular disease. Proc Natl Acad Sci U S A. 2006; 103:6970-6975. [PubMed: 16641093]

29. Furuhashi M, et al. Treatment of diabetes and atherosclerosis by inhibiting fatty-acid-binding protein aP2. Nature. 2007; 447:959-965. [PubMed: 17554340]

30. Sha RS, Kane CD, Xu Z, Banaszak LJ, Bernlohr DA. Modulation of ligand binding affinity of the adipocyte lipid-binding protein by selective mutation. Analysis in vitro and in situ. J Biol Chem. 1993; 268:7885-7892. [PubMed: 8463312]

31. Makowski L, Brittingham KC, Reynolds JM, Suttles J, Hotamisligil GS. The fatty acid-binding protein, aP2, coordinates macrophage cholesterol trafficking and inflammatory activity. 
Macrophage expression of aP2 impacts peroxisome proliferator-activated receptor gamma and IkappaB kinase activities. J Biol Chem. 2005; 280:12888-12895. Epub 12005 Jan 12831. [PubMed: 15684432]

32. Enoch HG, Catala A, Strittmatter P. Mechanism of rat liver microsomal stearyl-CoA desaturase. Studies of the substrate specificity, enzyme-substrate interactions, and the function of lipid. J Biol Chem. 1976; 251:5095-5103. [PubMed: 8453]

33. Heinemann FS, Ozols J. Stearoyl-CoA desaturase, a short-lived protein of endoplasmic reticulum with multiple control mechanisms. Prostaglandins Leukot Essent Fatty Acids. 2003; 68:123-133. [PubMed: 12538076]

34. Cao $\mathrm{H}$, et al. Identification of a lipokine, a lipid hormone linking adipose tissue to systemic metabolism. Cell. 2008; 134:933-944. [PubMed: 18805087]

35. Joseph SB, et al. Direct and indirect mechanisms for regulation of fatty acid synthase gene expression by liver X receptors. J Biol Chem. 2002; 277:11019-11025. [PubMed: 11790787]

36. Chu K, Miyazaki M, Man WC, Ntambi JM. Stearoyl-coenzyme A desaturase 1 deficiency protects against hypertriglyceridemia and increases plasma high-density lipoprotein cholesterol induced by liver X receptor activation. Mol Cell Biol. 2006; 26:6786-6798. [PubMed: 16943421]

37. Venkateswaran A, et al. Human white/murine ABC8 mRNA levels are highly induced in lipidloaded macrophages. A transcriptional role for specific oxysterols. J Biol Chem. 2000; 275:14700-14707. [PubMed: 10799558]

38. Repa JJ, et al. Regulation of absorption and ABC1-mediated efflux of cholesterol by RXR heterodimers. Science. 2000; 289:1524-1529. [PubMed: 10968783]

39. Joseph SB, et al. LXR-dependent gene expression is important for macrophage survival and the innate immune response. Cell. 2004; 119:299-309. [PubMed: 15479645]

40. Peet DJ, et al. Cholesterol and bile acid metabolism are impaired in mice lacking the nuclear oxysterol receptor LXR alpha. Cell. 1998; 93:693-704. [PubMed: 9630215]

41. Aqel NM, Ball RY, Waldmann H, Mitchinson MJ. Monocytic origin of foam cells in human atherosclerotic plaques. Atherosclerosis. 1984; 53:265-271. [PubMed: 6397199]

42. Han S, et al. Macrophage insulin receptor deficiency increases ER stress-induced apoptosis and necrotic core formation in advanced atherosclerotic lesions. Cell Metab. 2006; 3:257-266. [PubMed: 16581003]

43. Boord JB, et al. Adipocyte fatty acid-binding protein, aP2, alters late atherosclerotic lesion formation in severe hypercholesterolemia. Arterioscler Thromb Vasc Biol. 2002; 22:1686-1691. [PubMed: 12377750]

44. Listenberger LL, et al. Triglyceride accumulation protects against fatty acid-induced lipotoxicity. Proc Natl Acad Sci U S A. 2003; 100:3077-3082. [PubMed: 12629214]

45. Brown JM, et al. Inhibition of stearoyl-coenzyme A desaturase 1 dissociates insulin resistance and obesity from atherosclerosis. Circulation. 2008; 118:1467-1475. [PubMed: 18794388]

46. Rolph MS, et al. Regulation of dendritic cell function and T cell priming by the fatty acid-binding protein aP2. J Immunol. 2006; 177:7794-7801. [PubMed: 17114450]

47. Erbay E, Cao H, Hotamisligil GS. Adipocyte/macrophage fatty acid binding proteins in metabolic syndrome. Curr Atheroscler Rep. 2007; 9:222-229. [PubMed: 18241617]

48. Gregor MF, et al. Endoplasmic reticulum stress is reduced in tissues of obese subjects after weight loss. Diabetes. 2009; 58:693-700. [PubMed: 19066313]

49. Makowski L, Brittingham KC, Reynolds JM, Suttles J, Hotamisligil GS. The fatty acid-binding protein, aP2, coordinates macrophage cholesterol trafficking and inflammatory activity. Macrophage expression of aP2 impacts peroxisome proliferator-activated receptor gamma and IkappaB kinase activities. J Biol Chem. 2005; 280:12888-12895. [PubMed: 15684432]

50. Gomez FE, et al. Molecular differences caused by differentiation of 3T3-L1 preadipocytes in the presence of either dehydroepiandrosterone (DHEA) or 7-oxo-DHEA. Biochemistry. 2002; 41:5473-5482. [PubMed: 11969408] 
a.

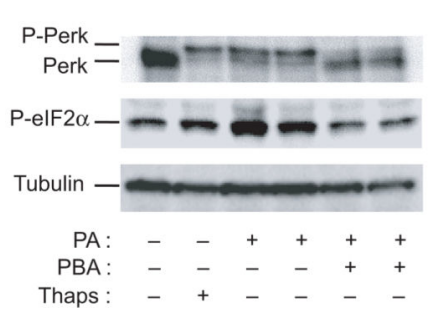

e.

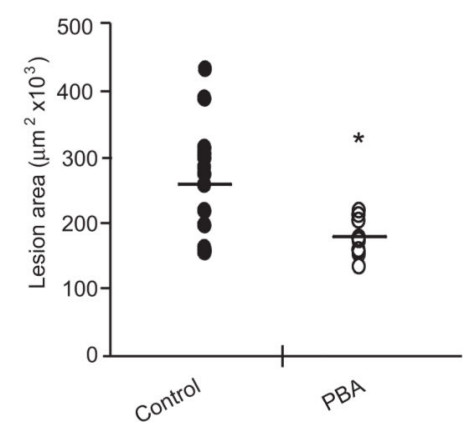

g.

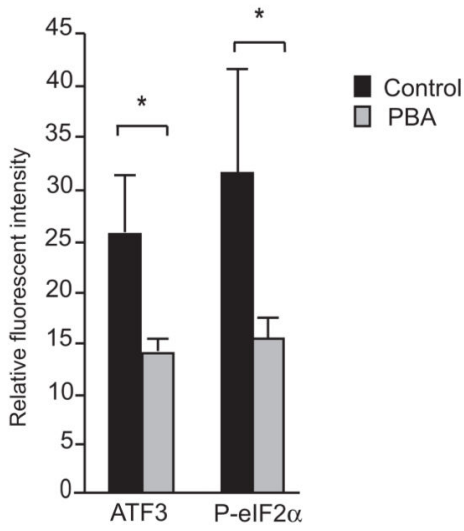

b.

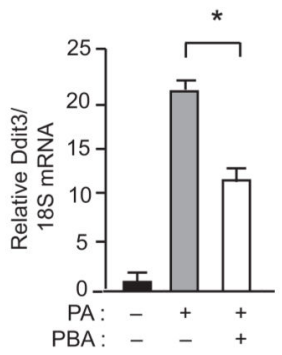

c.

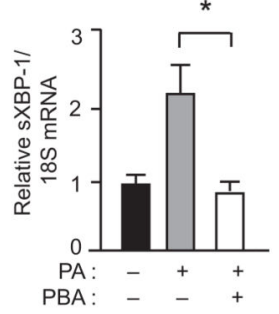

d.

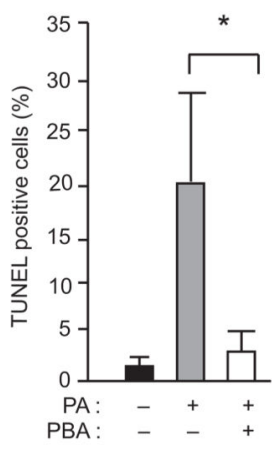

f.

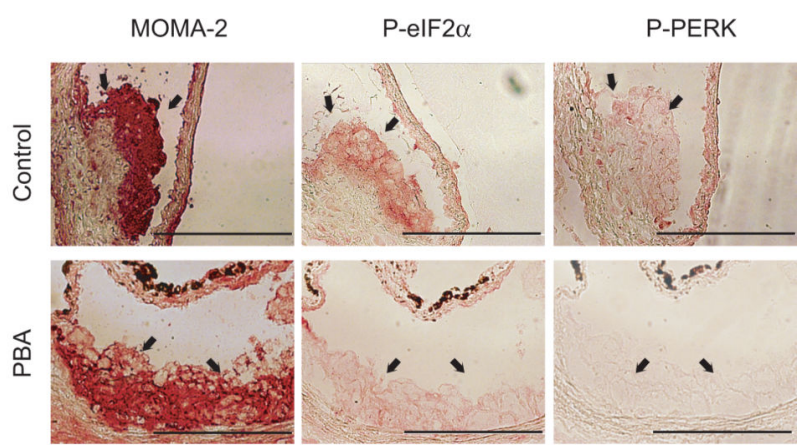

h.

Figure 1. PBA treatment protects against macrophage ER stress and reduces vascular disease progression

(a-d) We induced ER stress in WT macrophages by $500 \mu \mathrm{M}$ PA or $300 \mathrm{nM}$ thapsigargin (Thaps) in the presence or absence of $3 \mathrm{mM}$ PBA. P-PERK and P-eIF2-a were examined by Western blotting (a), mRNA levels of Ddit3 (b) and sXBP-1 were examined by qRT-PCR (c), and apoptosis were examined by TUNEL assays (d) (data represent mean \pm SEM; * $\mathrm{p}<0.05)$. ( $\mathbf{e}-\mathbf{g})$ Atherosclerotic lesion area in the aortic sinus from mice treated with either control or PBA for 2 weeks $(\mathrm{n} \geq 13)$ is reported (data represents mean $\pm \mathrm{SD}$; * indicates 
$\mathrm{p}<0.05)$ (e) and the serial sections were stained with antibodies against P-PERK (f), P-eIF2a (f), ATF3 (g) and MOMA-2 (f,g) (Red indicates positive staining with antibody. Scale bars represent $200 \mu \mathrm{m}$ ). (g) Relative fluorescent intensity was calculated for antibody staining corresponding to ATF3 and P- eIF2-a expression ( $\mathrm{n} \geq 3)$ in the macrophage-dense areas (data represent mean $\pm \mathrm{SD}$; * indicates $\mathrm{p}<0.05$ ). (h) Percent of apoptotic cells (TUNEL positive) in the aortic sinus area are shown for PBS (control) or $100 \mathrm{mg} \mathrm{kg}^{-1} \mathrm{PBA}$ (PBA-100) treated mice (Arrows point to apoptotic cells. Scale bars represent $200 \mu \mathrm{m}$. *indicates $\mathrm{p}<0.05)$. 
a.

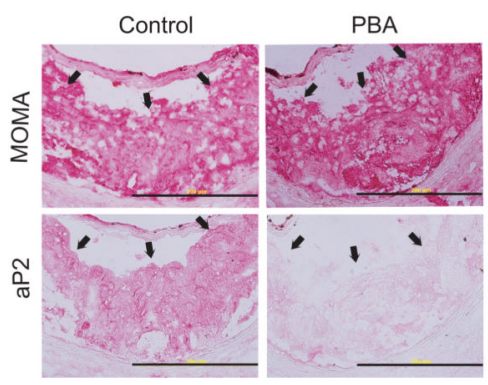

b.
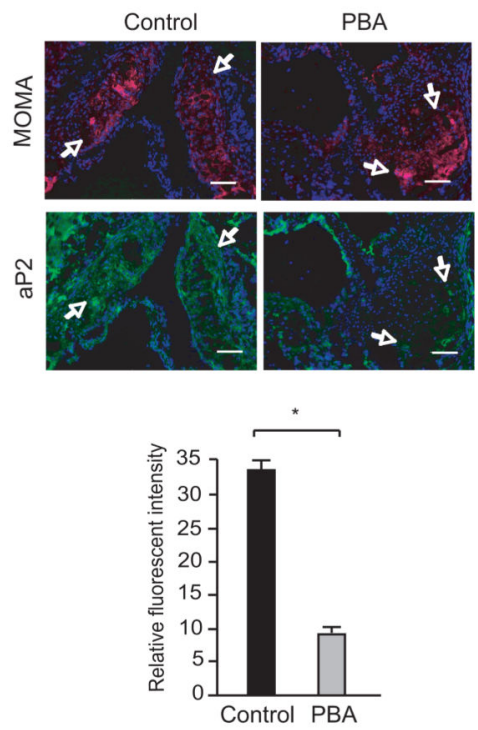

c.

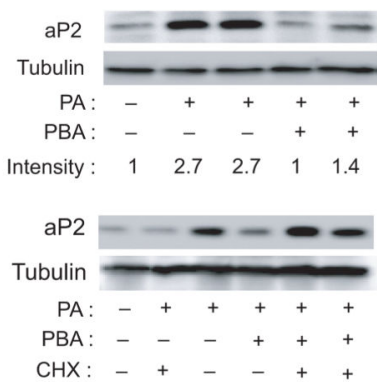

d.

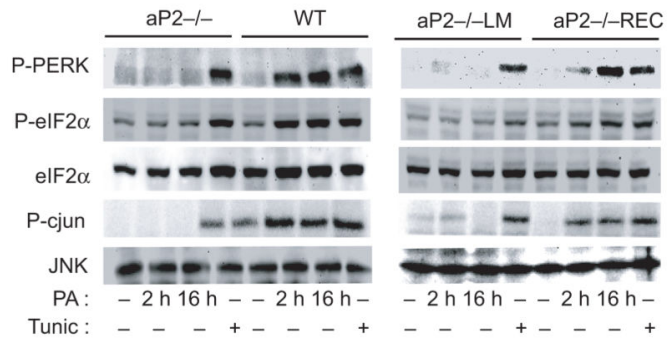

e.

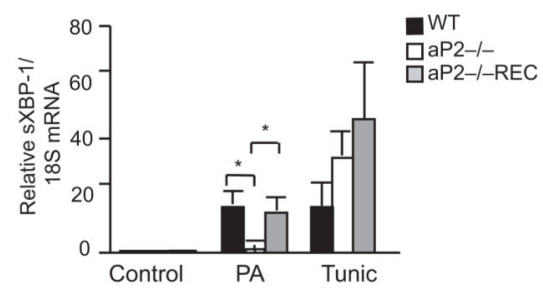

f.

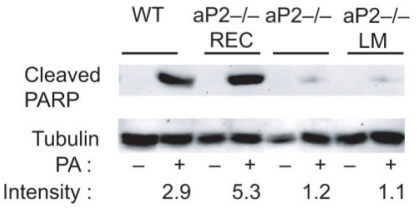

g.

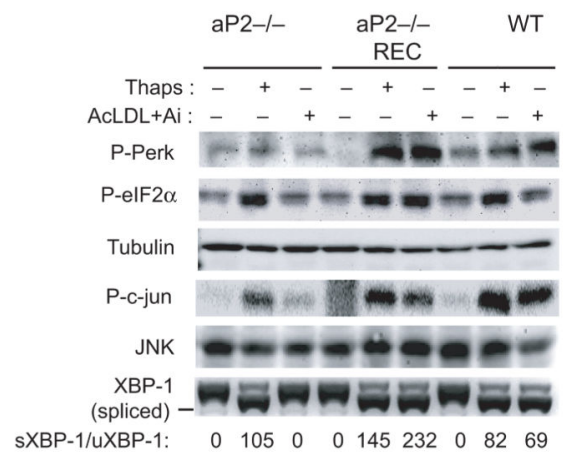

Figure 2. Requirement for aP2 in lipid-induced ER stress and apoptosis

(a-b) Serial sections from aortic sinus of PBS or PBA treated $A p o E^{-/-}$mice were stained for antibodies against MOMA-2 or aP2 (Arrows point to red staining for these antibodies in macrophage-dense areas. Scale bar represents $200 \mu \mathrm{m})$ (b) Relative fluorescence intensity for aP2 expression (green) in the macrophage-dense areas (red) of the lesions was calculated (Scale bar represents $100 \mu \mathrm{m}$. Data represent mean \pm SD; * indicates $\mathrm{p}<0.05$ and $\mathrm{n} \geq 3$ ). (c) aP2 expression was determined by Western blotting in PA $(500 \mu \mathrm{M})$ treated macrophages in the presence or absence of $3 \mu \mathrm{M}$ PBA or $100 \mu \mathrm{g} \mathrm{mL}^{-1}$ cycloheximide (CHX). (d) P-PERK, 
P-eIF2- $a$ was examined by Western blotting and JNK activity (P-cJun) by an in vitro kinase assay from PA $(500 \mu \mathrm{M})$ treated macorphages. (e) sXBP-1 was examined by qRT-PCR in $(500 \mu \mathrm{M})$ PA- or $(2 \mu \mathrm{g} / \mathrm{mL})$ tunicamycin (Tunic) treated $(12 \mathrm{~h})$ macrophages. (f) Cleaved PARP and tubulin expression were examined by Western blotting in PA-treated (24 h) macrophages (relative band intensities were quantified and data represent mean $\pm \mathrm{SEM}$; *p<0.05). (g) P-PERK, P-eIF2-a were examined by Western blotting, JNK activity (P-cJun) by a kinase assay, and $\mathrm{s} X B P-1$ by RT-PCR from macrophages treated with $10 \mu \mathrm{M}$ ACAT inhibitor (Ai) and $100 \mu \mathrm{g} / \mathrm{mL}$ Ac-LDL (24 hours) or $300 \mathrm{nM}$ thapsgiargin (Thaps) (The ratio of relative intensities corresponding to spliced (s-XBP1) and unspliced ( $\mathrm{u}-\mathrm{XBP} 1$ ) were calculated). 

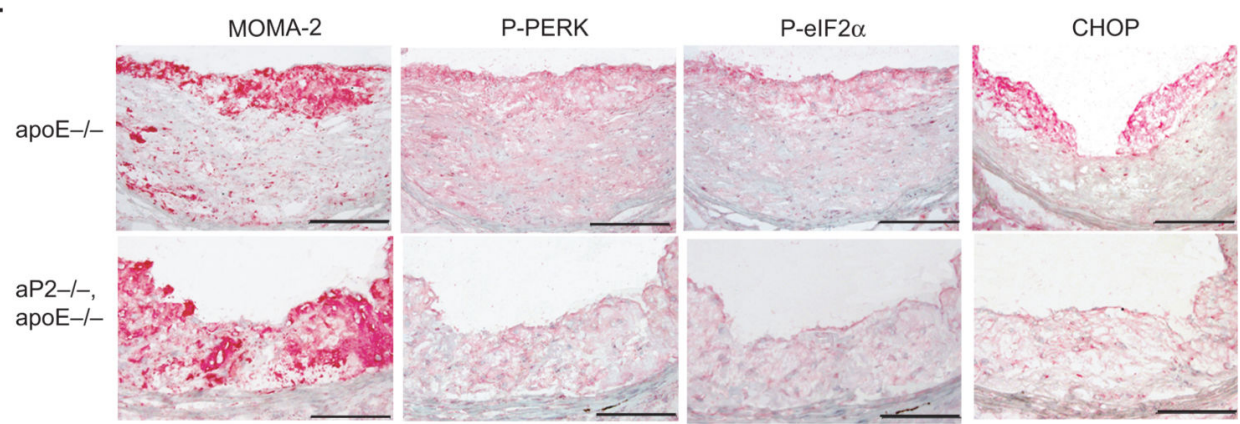

b.

MOMA-2

ATF3
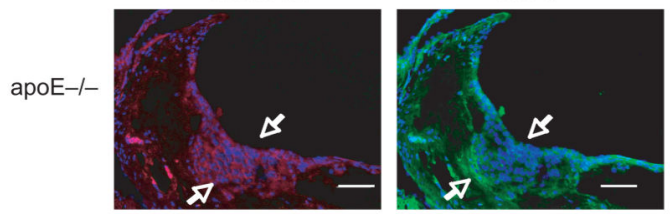

MOMA-2

P-elF20
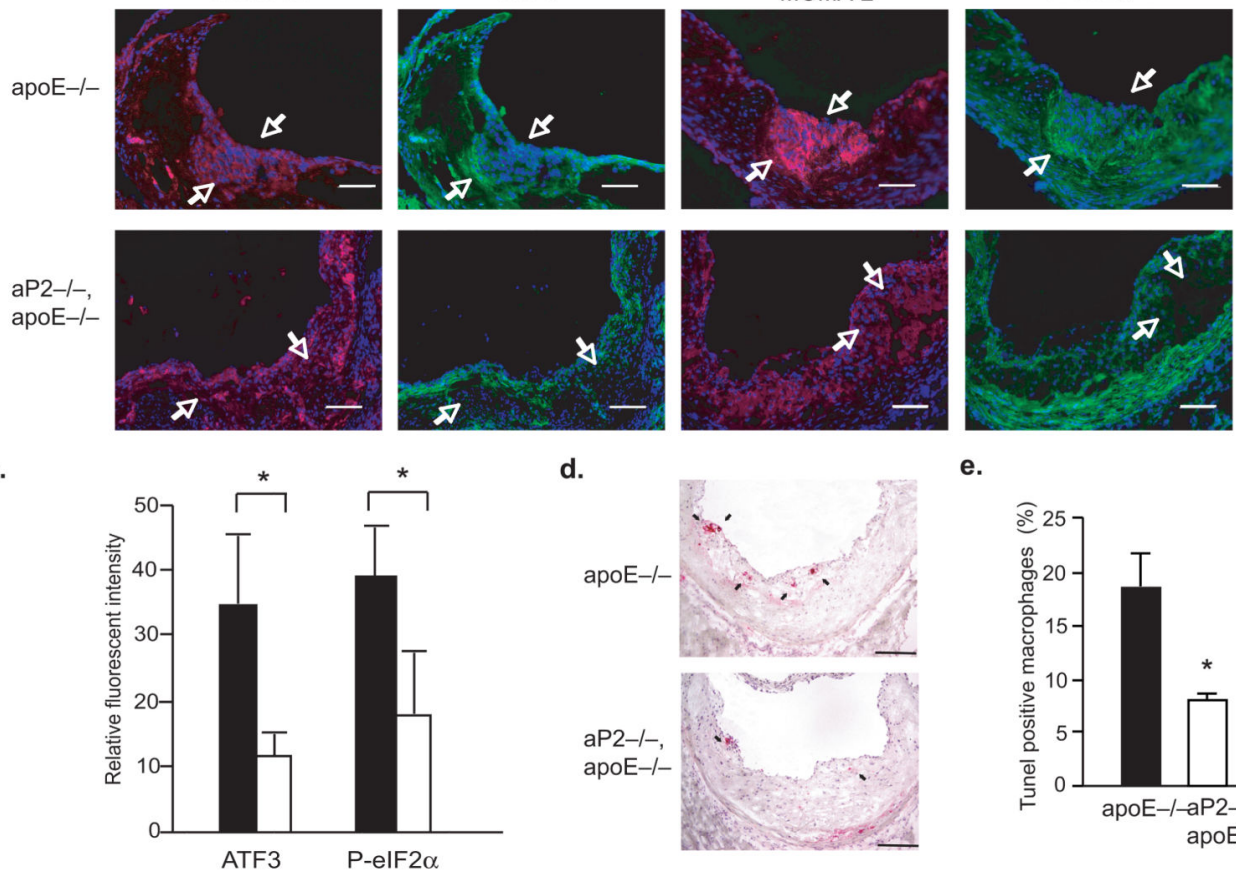

d.
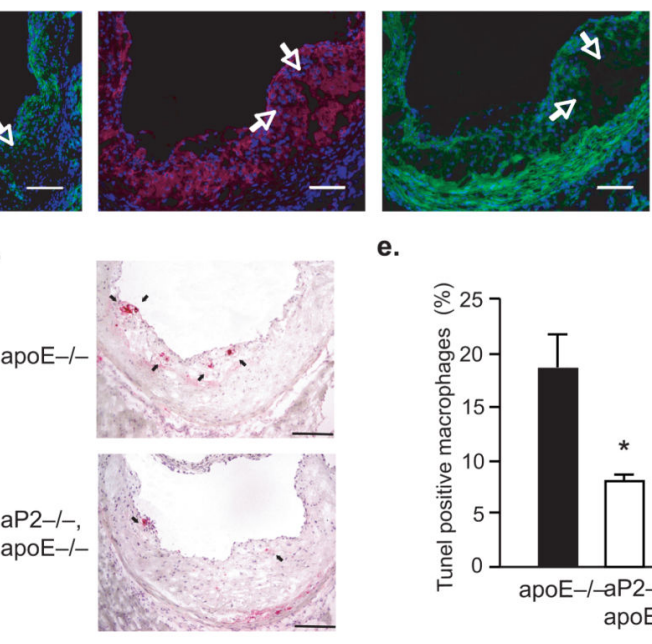

e.

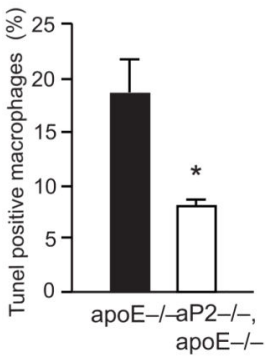

f.

g.

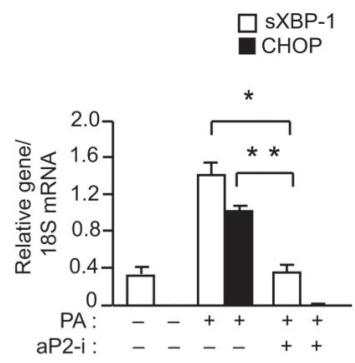

h.

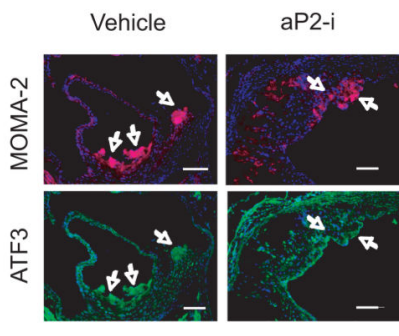

Figure 3. aP2 deficiency protects from hypercholesteremia induced macrophage ER stress and apoptosis in atherosclerotic lesions

(a-b) Immunohistochemical staining with antibodies against MOMA-2, P-PERK, P-eIF2-a (a,b), CHOP (a), and ATF3 (b) were performed in atherosclerotic lesions from the proximal aorta of $A p o E^{-/-}$and $a P 2^{-/-} A p o E^{-/-}$mice fed a Western diet for 16 weeks (Arrows point to ATF3 and P-eIF2-a (green), expressed in the MOMA-2 positive (red) areas of the lesions. Scale bars in (a) represent $50 \mu \mathrm{m}$ and in (b) represent $100 \mu \mathrm{m}$ ). (c) Relative fluorescent intensity was calculated for stainings corresponding to ATF3 and P-eIF2-a in 
the macrophage-dense areas (data represent mean $\pm \mathrm{SD} ; *$ indicates $\mathrm{p}<0.05, \mathrm{n} \geq 3$ ). (d-e)

Apoptotic macrophages in the lesions from $\mathrm{ApoE}^{-/-}$and $\mathrm{aP}^{-/-} \mathrm{ApoE}^{-/-}$mice were determined by TUNEL assay (Arrows point to apoptotic cells. Scale bars represent $100 \mu \mathrm{m}$.

* indicates $\mathrm{p}<0.05)$. (f-g) Macrophage lines were stressed with PA $(500 \mu \mathrm{M})$ in the presence of vehicle (-) or varying does of the aP2-i $(0.1-50 \mu \mathrm{M})$. P-PERK and P-eIF2- $\alpha$ was examined by Western blotting (f) and $\mathrm{s} X B P-1$ and $D d i t 3$ mRNA were analyzed by qRT-PCR from macrophages treated with $25 \mu \mathrm{M}$ of aP2-i (g). (h) Double immunofluorescent staining was performed using antibodies against MOMA-2 and ATF-3 in the atherosclerotic lesions from $A p o E^{-/-}$mice treated with vehicle or aP2-i ( $15 \mathrm{mg} \mathrm{kg}^{-1}$ for 14 weeks) (Arrows indicate staining for ATF3 (green) in MOMA-2 positive areas (red). Scale bars represent $100 \mu \mathrm{m})$. 
a.

TG

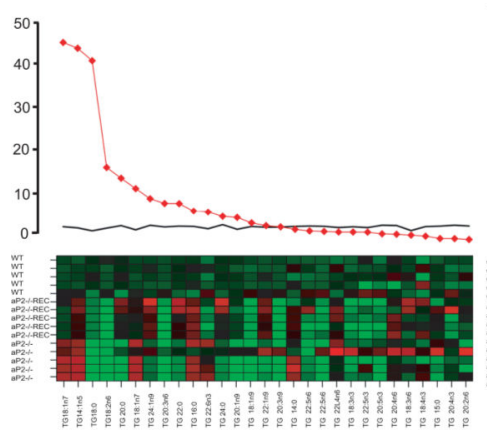

$\mathrm{PL}$
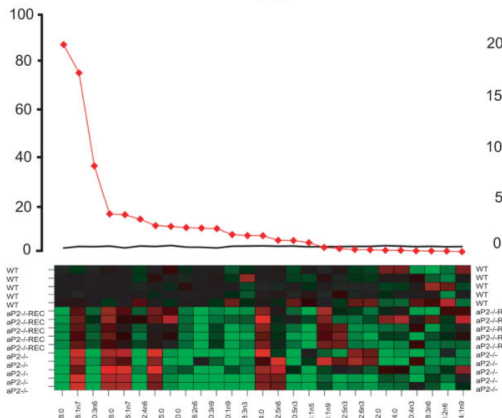
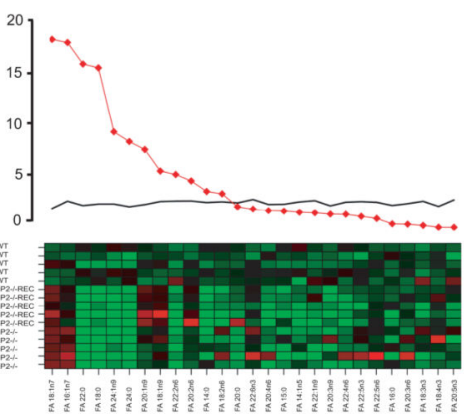

b.
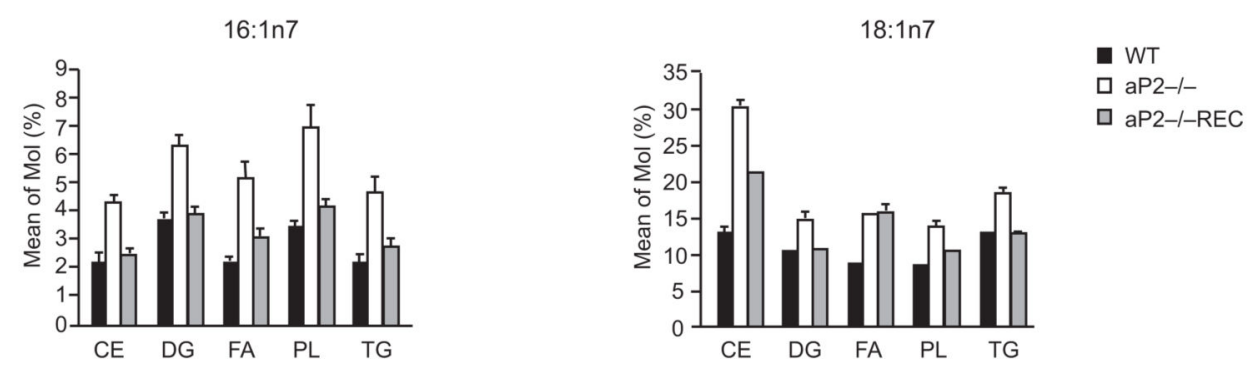

c.

d.
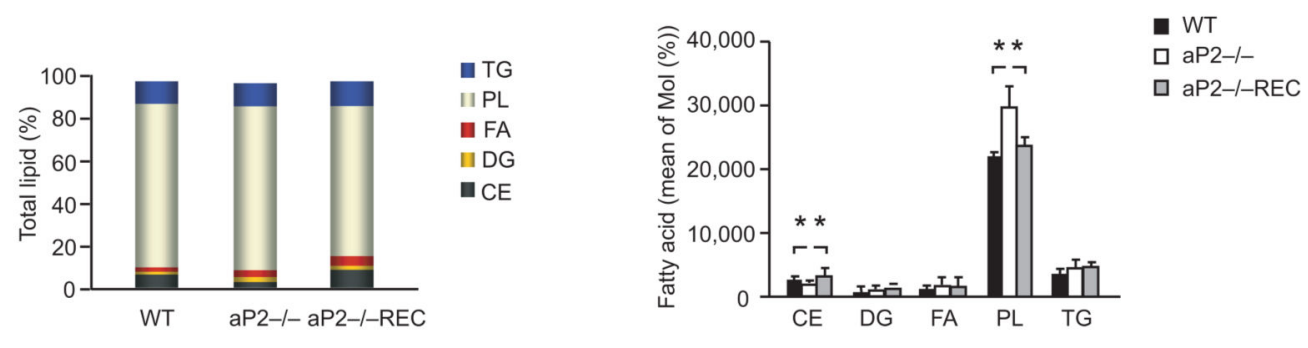

Figure 4. Regulation of macrophage lipid composition by aP2

(a) Lipid class composition analysis for TG, PL and FA was performed. The F statistics from one-way ANOVA are displayed as red diamonds over the distribution of F statistics from permuted data. The black line is the $95^{\text {th }}$ percentile of the F statistics over 1000 permutations. The higher the value of the F-statistics from ANOVA, the more different the groups are. The heat map displays the observed data, centered to the mean of the WT genotype and scaled by the standard deviation of all observations. (b) The mean concentration of $\mathrm{C} 16: 1 \mathrm{n} 7$ and $\mathrm{C} 18: 1 \mathrm{n} 7$ was determined for each lipid class in the various 
macrophage lines. (c-d) Total lipid composition: Percent total lipids (c) and bar plots of the mean concentration of lipids (d) in the macrophage lines. 
a.

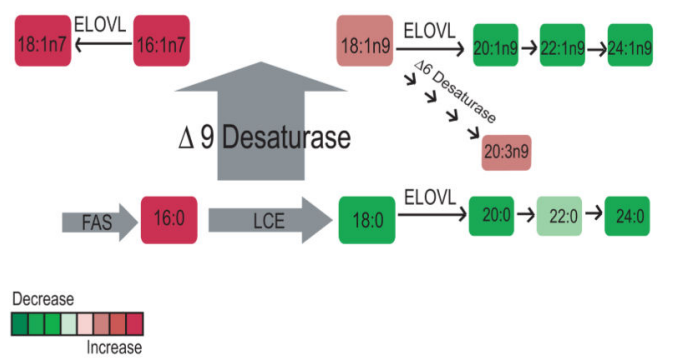

d.

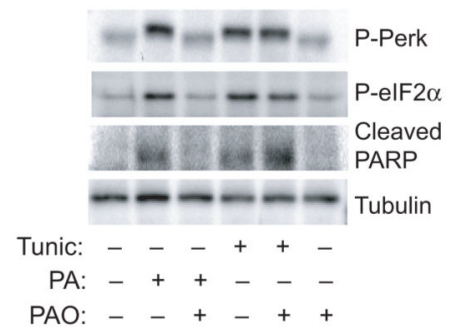

f.

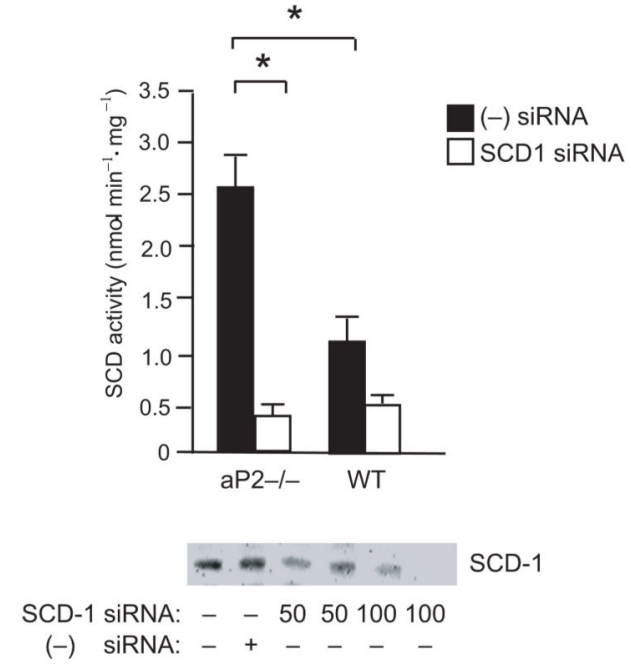

b.

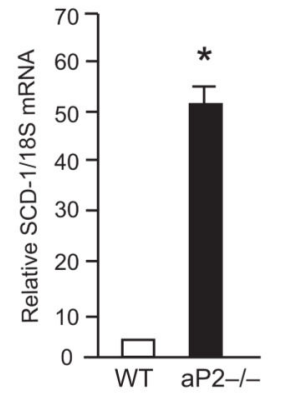

c.

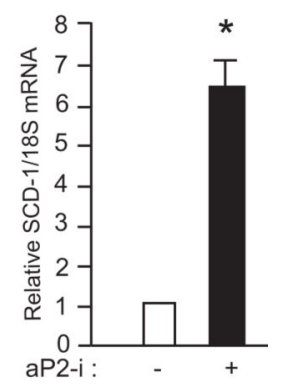

e.

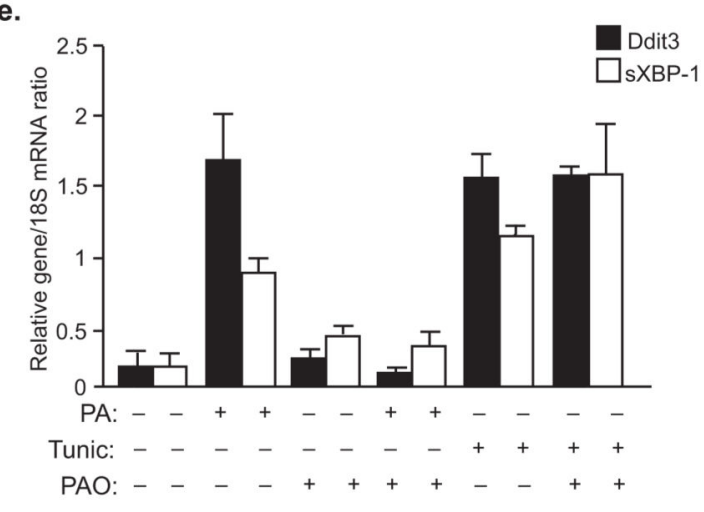

g.

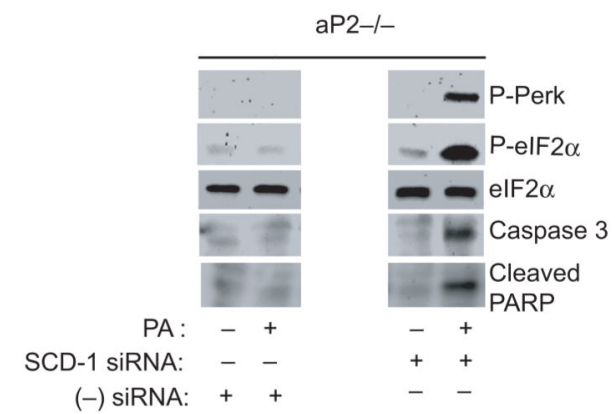

Figure 5. A central role for SCD and C16:1n7 in aP2 mediated lipotoxic signaling

(A) A summary of the lipid changes that occur as a result of aP2 deficiency in macrophages (LCE and ELOVL; fatty acid elongase for long chain fatty acid). (b)SCD-1 mRNA levels were examined by qRT-PCR in primary peritoneal macrophages at the base line or (c) after treatment of animals aP2-i for 6 weeks $(n=6)$ (data represent mean \pm SEM; * indicates $\mathrm{p}<0.05)$. (d-e) ER stress was induced in macrophages by PA $(300 \mu \mathrm{M})$ or tunicamycin $(2$ $\mathrm{mM})$ treatment for 3 hours. Cells were pretreated with PAO $(300 \mu \mathrm{M})$ PAO, where indicated. P-PERK, P-eIF2- $a$ and cleaved PARP were examined by Western blotting (d) 
and Ddit3 and sXBP-1 mRNA were examined by qRT-PCR (E). (F) From macrophage lines treated with SCD-1 siRNA (50-100 nM) or scrambled (-) siRNA, SCD activity (upper panel) was examined by an enzymatic assay and SCD protein expression (lower panel) was examined by Western blotting (G) P-PERK, P-eIF2- $a$ and cleaved PARP were examined by Western blotting from lysates of $a 2^{-/-}$macrophages treated with negative (-) siRNA or SCD-1 specific siRNA $(100 \mathrm{nM})$ and treated with or without PA $(500 \mu \mathrm{M})$ (data represent mean \pm ; SEM; * indicates $\mathrm{p}<0.05)$. 
a.

e.

g.

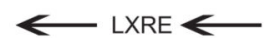

Fasn : $5^{\prime}$ - gcga TGACCG gcag TAACCC cggc $-3^{\prime}$ SCD-1: $5^{\prime}$ - agcg TGACCA cagg TAACCT caac $-3^{\prime}$ b.

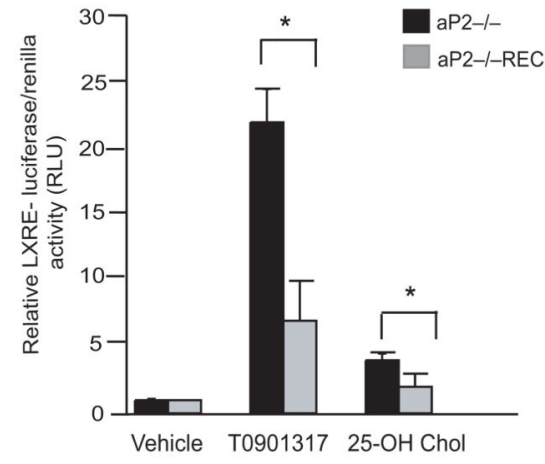

c.

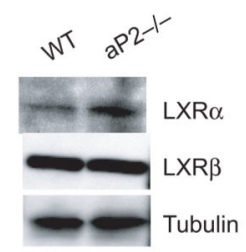

d.
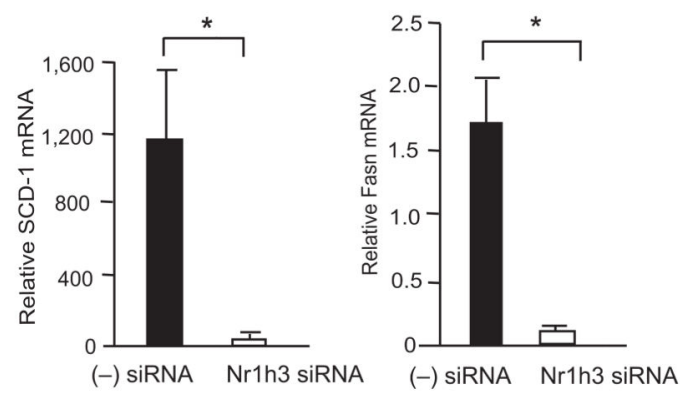

f.

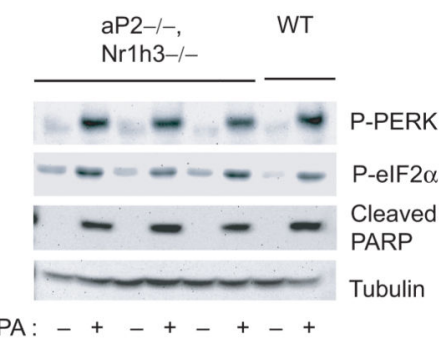

$-+-+-+$

$\mathrm{PA}:-+\ldots+\ldots+\ldots+$

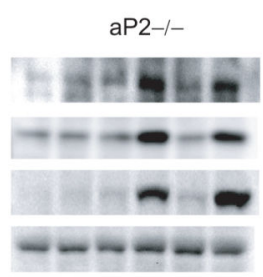

$\mathrm{PA}:-+-+-+$
(-) $\operatorname{siRNA:}++--\ldots$

Nr1h3 SiRNA: $-{ }_{-}++-$

SCD1 SiRNA:

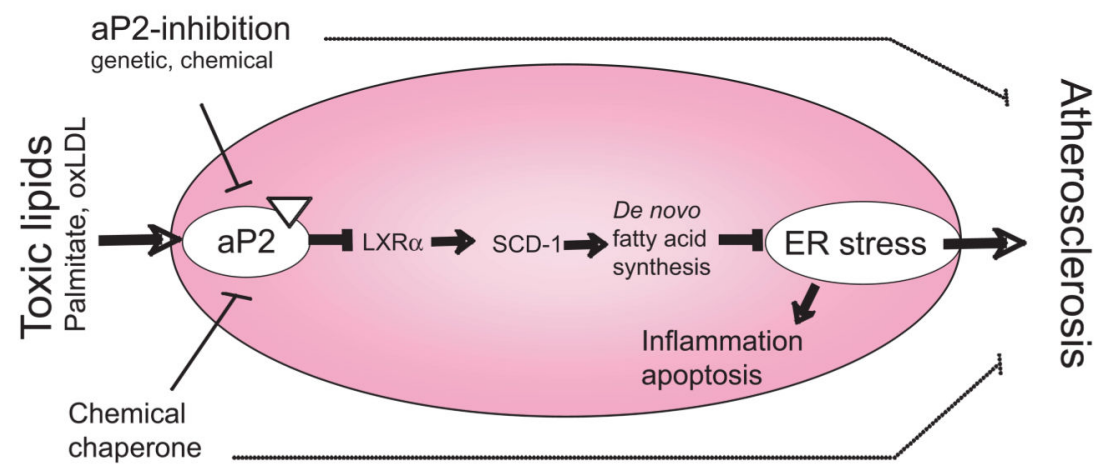

Figure 6. Linking toxic lipids to ER stress and atherosclerosis through aP2-LXR-a crosstalk (a) Alignment of LXR responsive element (LXRE) on Fasn and SCD-1 promoters. (b) LXR-driven transcriptional activity was determined from various macrophage lines upon stimulation with a synthetic T0901317 $(10 \mu \mathrm{M})$ or endogenous 25 -hydroxycholesterol (10 $\mu \mathrm{M})$ LXR ligand (luciferase activity is reported after normalizing to transfection efficiency). (c) Relative LXR- $\alpha$ and LXR- $\beta$ protein levels in various macrophage lines were examined by Western blotting (d) Relative SCD-1 and Fasn mRNA levels from $a 2^{-/-}$macrophage treated with a scrambled (-) or NrIh3-specific siRNA were examined by qRT-PCR. (e) 
Lysates from various macrophages treated with scrambled (-) siRNA or a specific siRNA against $S C D-1$ or $N r l h 3$ and stressed with or without PA $(500 \mu \mathrm{M})$ were examined for PPERK and P-eIF2-a by Western blotting. (f) Lysates from peritoneal macrophages from $a P 2^{-/-} \mathrm{NrIh}^{-/-}$or WT mice stressed with or without PA $(500 \mu \mathrm{M})$ were examined for PPERK, P-eIF2-a and cleaved PARP by Western blotting. (g) A cellular lipotoxicity model: Toxic levels of lipids are sensed by the ER through an aP2-dependent pathway and induce the UPR and lead to macrophage apoptosis. The absence of aP2 serves to reactivate macrophage de novo lipogenesis pathways and promotes desaturation, particularly through LXRa-mediated activation of SCD-1, leading to increased production of bioactive lipids and resistance to ER stress. Our findings indicate that alleviation of macrophage ER stress, either through aP2 inhibition or enhancing ER function, is protective against atherosclerosis. 